\title{
Significados dos episódios maníacos para pacientes com transtorno bipolar em remissão: um estudo qualitativo
}

\author{
Meanings of manic episodes in patients with bipolar \\ disorder in remission: a qualitative study \\ Luiz Carlos Pereira Bin', Lia Keuchguerian Silveira Campos’', Amilton dos Santos Junior', Egberto Ribeiro \\ Turato ${ }^{1}$
}

\section{RESUMO}

Objetivo: Discutir os significados da vivência de episódios maníacos para pacientes com transtorno bipolar (TAB). Métodos: Trata-se de uma pesquisa qualitativa, feita por meio de entrevistas semidirigidas em profundidade, em uma amostra fechada pelo critério de saturação com oito pacientes com TAB em remissão. A técnica de tratamento de dados foi feita por meio da análise de conteúdo das entrevistas transcritas na íntegra e categorização. Os resultados foram submetidos à validação externa, no Laboratório de Pesquisa Clínico-Qualitativa do Departamento de Psicologia Médica e Psiquiatria da Unicamp, composto por 37 pesquisadores do método, entre eles mestrandos, doutorandos, pós-doutorados e pesquisadores seniores. Resultados: Foram identificadas três categorias - Ambivalência e vergonha: pensar ou não pensar sobre os episódios maníacos; Organizando sentimentos pessoais: a remissão como um momento de autoconsciência; Episódios maníacos estruturando relações interpessoais versus projeções da angústia. Conclusão: Os achados da presente pesquisa contribuem para a maior compreensão dos quadros maníacos no TAB, que podem auxiliar nas reflexões acerca da relação profissional-paciente, para elaborar estratégias para aderência

\section{Palavras-chave}

Transtorno bipolar, mania, assistência ao paciente, pesquisa qualitativa. e para as medidas terapêuticas e preventivas da recorrência dos episódios. Podem auxiliar a equipe de saúde envolvida no acompanhamento desses casos e também os pesquisadores na investigação da contribuição dos significados aqui discutidos nos fenômenos de aderência ao tratamento e de um melhor prognóstico.

\section{ABSTRACT}

Objective: To discuss the meanings of the experiences of manic episodes in patients with bipolar disorder (BD). Methods: This is a qualitative research, done through semi-structured in-depth interviews in a sample closed by saturation criterion with eight patients with BD in remission. The technical data processing was done through qualitative content analysis of the transcribed interviews and categorization. The results were validated by peer-reviewers from the Laboratory of Clinical-Qualitative Research of the Department of Medical Psychology and Psychiatry, Unicamp consisting of 37 researchers of the method, including, masters,

1 Universidade Estadual de Campinas (Unicamp), Faculdade de Ciências Médicas, Departamento de Psicologia Médica e Psiquiatria, Laboratório de Pesquisa Clínico-Qualitativa (LPCQ).

Recebido em

2/1/2014

Aprovado em

$3 / 5 / 2014$

DOI: $10.1590 / 0047-2085000000018$
Endereço para correspondência: Lia Keuchguerian Silveira Campos

Av. José Rocha Bonfim, 214, Ed. Sidney,

sala 223, Jardim Santa Genebra

13080-650 - Campinas, SP, Brasil

Telefone: (19) 3255-0691

E-mail: liacampos81@uol.com.br 


\section{Keywords}

Bipolar disorder, mania, patient care, patient dropouts, qualitative research. doctoral, postdoctoral and senior researchers. Results: Three categories were identified Ambivalence and shame: to think or not to think about the manic episodes; Organizing personal feelings: remission as a moment of self-consciousness; Maniac episodes: structuring interpersonal relationships versus projections of self-criticism. Conclusion: The findings of this research contribute to a greater understanding of manic frames in BD that can assist in the reflections on the doctor-patient relationship, to develop strategies for adherence and the therapeutic and preventive measures of recurrent. Can assist the health team involved in monitoring these cases and also the researchers in investigation of the contribution of these meanings discussed here in the phenomena of adherence to treatment and a better prognosis.

\section{INTRODUÇÃO}

O transtorno afetivo bipolar (TAB) é um transtorno do humor caracterizado por alterações afetivas persistentes que podem durar semanas ou meses, com recorrência periódica ou cíclica', o que faz com que os pacientes não possam prever seus próprios estados emocionais.

Há dois padrões básicos de sintomas: a fase depressiva, caracterizada por humor depressivo e redução da energia e da atividade²; e a fase maníaca, caracterizada pelo estado de humor elevado e aumento da energia e da atividade ${ }^{2}$. A mania é o mais característico dos episódios, além de frequente e incapacitante ${ }^{3}$. Essa fase tem uma particularidade diferente da fase depressiva, na qual o paciente sente o incômodo de sua condição; durante o episódio de mania, ele pode não se perceber doente. A crítica fica prejudicada e os ajuizamentos emitidos ficam afastados da realidade do paciente ${ }^{3}$. De acordo com os estudos de Silva et al. ${ }^{4}$, pacientes com TAB em mania avaliam de forma não fidedigna seu estado afetivo.

De acordo com Souza5, a eutimia é definida como a remissão dos sintomas e seria o período no qual o paciente estaria reintegrado funcionalmente em suas atividades de rotina. O tratamento do paciente eutímico deve considerar a possibilidade de ele vir a ter episódios de mania e/ou depressão $0^{5}$. Em um estudo de evolução de longo prazo do TAB, constatou-se que o número total de meses em eutimia se correlacionou negativamente com a idade do paciente e a duração do transtorno; os participantes da pesquisa apresentaram evolução desfavorável com longos períodos sintomáticos e importante comprometimento sócio-ocupacional.

O TAB é acompanhado por risco aumentado de tentativas de suicídio e de suicídio consumado ${ }^{7}$, confirmando a importância de estudos que busquem entender todas as suas dimensões. Entre 14\% e 59\% dos pacientes apresentam ideação suicida e entre 25\% e 56\% têm pelo menos uma tentativa de suicídio ao longo da vida. Aproximadamente, 15\% a 19\% dos pacientes com TAB morrem por suicídio.

Caycedo et al. ${ }^{8}$ constataram que o diagnóstico de maior prevalência em relação ao risco de recaída é o TAB. No estudo de Moreno et al. ${ }^{3}$, destacou-se que a mania é a que mais resulta em internações agudas em virtude das graves mudanças de comportamento e conduta que provoca.

Eventos estressantes, como divórcio ou separação, problemas no trabalho ou interpessoais e doença, têm sido associados ao TAB'. A identificação desses eventos pode ser importante para a clínica, por necessitar de maior atenção no tratamento considerando o constatado risco de recaída?.

Com base no que foi discutido, percebeu-se uma lacuna na literatura em relação aos significados que são atribuídos pelos pacientes ao TAB, com a hipótese de que o conhecimento desses significados poderia então contribuir de forma significativa para maior compreensão do transtorno e auxiliar nos processos de adesão ao tratamento, diminuição das recaídas e melhora no prognóstico. A presente pesquisa tem como objetivo discutir os significados dos episódios maníacos para pacientes com diagnóstico de TAB, relatados durante a fase de remissão.

\section{MÉTODOS}

O presente estudo utilizou o método qualitativo, tal como concebido com base na abordagem humanística, aqui aplicado a um setting assistencial em saúde. Nos settings da saúde, as pesquisas qualitativas possibilitam conhecer as significações dos fenômenos do processo saúde-doença para melhorar a qualidade da relação profissional-paciente-família-instituição, promover maior adesão de pacientes e da população a tratamentos individuais e de medidas implementadas coletivamente, além de possibilitar a compreensão dos sentimentos, ideias e comportamentos dos acometidos por doenças, assim como de seus familiares e da equipe profissional de saúde envolvida10,11.

Para a condução da presente pesquisa, foi feita a apresentação de seus participantes pela equipe médica do Ambulatório de Psiquiatria Geral de Adultos do Hospital das Clínicas da Universidade Estadual de Campinas (HC-Unicamp), um hospital terciário, localizado na cidade de Campinas, estado de São Paulo, na região sudeste do Brasil. Foram incluí- 
dos na amostra pacientes adultos que se encontravam sob seguimento ambulatorial e que receberam o diagnóstico de $T A B$; todos em fase de remissão com história pregressa de pelo menos uma fase maníaca, conforme confirmado pela equipe médica.

A amostra, composta por oito participantes (Tabela 1), foi intencional e fechada pelo critério de saturação ${ }^{12}$, ou seja, após a realização das entrevistas, não havia adição de dados substancialmente novos na avaliação dos pesquisadores e de seus pares do Laboratório de Pesquisa Clínico-Qualitativa do Departamento de Psicologia Médica e Psiquiatria da Faculdade de Ciências Médicas da Unicamp. Importante ressaltar que esses dados foram submetidos à validação externa pelos pares acadêmicos do respectivo grupo de pesquisa da universidade.

Tabela 1. Caracterização da amostra segundo gênero, idade, estado civil, número de filhos e denominação religiosa

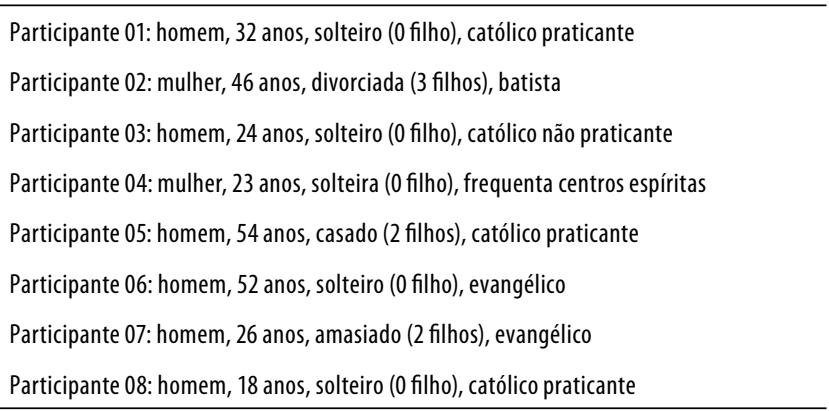

A coleta de dados foi realizada pelo primeiro autor do presente artigo. Cuidados visando ao rigor metodológico das entrevistas foram tomados, assim como a supervisão e orientação do entrevistador durante todo o processo. Esses cuidados técnicos foram: facilitar a livre expressão do entrevistado; boa relação estabelecida entre entrevistador e entrevistado; local de entrevista privativo; adoção do sistema de peer-review nas etapas de elaboração do projeto e da discussão dos resultados obtidos no Laboratório de Pesquisa Clínico-Qualitativa.

Entrevistas semidirigidas de questões abertas foram empregadas $^{10,13}$ a fim de obter o relato das experiências de vida dos participantes para encontrar os significados psicológicos atribuídos aos episódios maníacos. Para isso, essas entrevistas foram feitas em profundidade, com duração de 1 a 2 horas, e os entrevistados foram estimulados a expressar suas considerações a respeito do tema de estudo em uma fala espontânea sobre suas ideias, a fim de manter a fluidez na entrevista ${ }^{10,13}$.

Após estabelecimento do rapport e leitura explicativa do termo de consentimento livre e esclarecido, as entrevistas começavam com a pergunta disparadora "Você poderia me contar como foram aquelas fases de grande euforia?" Essa questão era complementada, caso não tivesse sido mencionado espontaneamente, com o convite para falar sobre os seguintes subtemas: modo de lidar com os sentimentos e os pensamentos advindos da vivência do episódio maníaco; eventuais hospitalizações; reações emocionais diante da possibilidade de recaída; como os episódios vivenciados afetavam suas relações interpessoais atuais.

Todas as entrevistas foram registradas em gravador de voz e transcritas posteriormente. Para atingir uma interpretação profunda dos dados coletados, desenvolveu-se a análise qualitativa de conteúdo ${ }^{14}$.

Por meio de rigoroso processo de reflexão e associações com teorias já publicadas na literatura, os autores realizaram leituras flutuantes do conjunto das entrevistas ${ }^{10}$, em busca de núcleos de sentido para emergir categorias de discussão. Essas categorias são formadas de acordo com os conteúdos latentes dos discursos que eram associáveis entre si. As categorias na análise qualitativa não são formadas com base na frequência das unidades de análise ou de outras abordagens matemáticas, o raciocínio é decorrente da identificação dos fenômenos associados com as respostas dos entrevistados, respeitando o caráter êmico ${ }^{13}$, isto é, a construção de uma discussão na perspectiva dos sujeitos entrevistados. Os fenômenos assim identificados podem então ser interpretados de modo a gerar conceitos capazes de ser generalizados ${ }^{14}$.

Cuidados éticos foram tomados seguindo as normas preconizadas pelo Conselho Nacional de Saúde. Os participantes assinaram o termo de consentimento livre e esclarecido, permitindo que suas falas fossem transcritas e divulgadas anonimamente. O projeto de pesquisa foi aprovado pelo Comitê de Ética em Pesquisa da Faculdade de Ciências Médicas da Unicamp, em 22 de outubro de 2008, protocolo 861/2008.

\section{RESULTADOS}

No processo de interpretação dos dados da presente pesquisa, foram encontrados muitos aspectos que poderiam compor categorias, e entre eles foram enfatizados aqueles que compuseram três categorias, que serão apresentadas neste artigo por atenderem ao objetivo comum de buscar maior compreensão dos significados dos episódios maníacos para pacientes com TAB. São elas: (1) Ambivalência e vergonha: pensar ou não pensar sobre os episódios maníacos; (2) Organizando sentimentos pessoais: a remissão como um momento de autoconsciência; (3) Episódios maníacos estruturando relações interpessoais versus projeções da angústia. Vinhetas das entrevistas foram utilizadas para ilustrar os conteúdos discutidos.

\section{(1) Ambivalência e vergonha: pensar ou não pensar sobre os episódios maníacos}

Com base nos achados, identificaram-se dois tipos de reações dos entrevistados quando estimulados a falar sobre os 
episódios maníacos: relatavam com muitos detalhes as suas histórias pregressas, demonstrando crítica ao comportamento que tiveram, ou diziam simplesmente não se lembrar dos fatos ocorridos durante esses episódios.

"(...) eu entrei na casa de um pessoal, sem noção nenhuma, chutei a porta. Eu não sei... Como foi incrível... Eu invadi a casa da mulher... Eu estava sem noção (...)" (P7)

"Não lembro muita coisa, não lembro o que fiz." (P1)

Pode-se entender que esse contraste de posturas não configura dois pontos isolados. Há um continuum que vai de um aparente esquecimento até posturas muito críticas a respeito do que ocorreu.

Ao longo das entrevistas, ao serem instigados a contar suas histórias, revelam fatos isolados com um discurso muitas vezes desconexo, banalizando algumas condutas claramente decorrentes do estado maníaco.

"Não... A fase de euforia não foi uma fase assim, de... Os maiores problemas foram a ansiedade e eu não ter sono pra dormir." (P5)

"Porque eu estava cansado, sabe? Era muita zoeira, era véspera de natal, feriado... Aí fiquei doido... Aí atacou o surto." (P6)

Os dados sugerem relutância em lembrar-se do episódio maníaco, o que pode representar uma confusão de sentimentos. Há, em alguns momentos, a utilização de um mecanismo de negação, a fim de ignorar ou banalizar a exaltação de humor característica das fases maníacas, racionalizando-as com justificativas pautadas em questões fisiológicas ou exacerbação de situações cotidianas.

\section{"... quando uma pessoa tem um pensamento que é fora da realidade, talvez isso tenha um motivo para ela, entendeu? (...) talvez aquilo preencha um buraco assim, talvez uma necessidade." (P4) \\ "(...) uma vez eu saí do banheiro, estava somente com a toalha e passei perto de um monte de gente que tinha lá em casa. Ah, fazia muitas coisas que até nem lembro..." (P2)}

Aparece uma crença de que os conteúdos dos episódios maníacos poderiam refletir alguma necessidade pessoal. Assim sendo, aparece a vergonha como um sentimento que levaria à supressão dos fatos.

\section{(2) Organizando sentimentos pessoais: a remissão como um momento de autoconsciência}

Os episódios maníacos com os sentimentos conflitivos e ambivalentes que resultam em vivências de vergonha podem também ser, após a reorganização do pensamento na fase de remissão, pontos de partida para uma postura de reavaliação da vida e tomada de consciência. Do ponto de vista psicodinâmico, o próprio episódio depressivo que segue o maníaco pode ser decorrente de uma atitude de autocrítica muito violenta do sujeito em relação àquilo que fez.

Durante as entrevistas, os informantes relatavam detalhadamente a intensa crítica ao comportamento inadequado durante a fase de mania, o que pode ser entendido como uma tentativa de organizar seus sentimentos e atitudes.

"Ah, que eu não sou uma pessoa igual aos outros, que
eu poderia levar minha vida numa boa, estar de bem
com a vida hoje, ter minhas coisas, já perdi tanta coi-
sa por causa dessa doença minha..." (P1)
"Eu acho que é legal ser uma pessoa normal né?
Agora, ser diferente, sei lá... Ser taxado como doente
mental não é fácil não." (P2)

Aparece nos relatos um estigma que, concomitantemente com as limitações impostas pelo transtorno, podem levar a sofrimento mental e a exacerbar a autocrítica. Surgem, por consequência, angústia e sentimentos de impotência, tristeza e dificuldades em relação ao tratamento.

"Mas parece que é a mesma coisa de eu estar lá dentro [internado], porque eu não posso sair sozinho, tenho que continuar tomando remédio." (P8)

Em virtude dessa estigmatização, os relatos revelam medo de estar alegre e essa alegria ser entendida como recaída ou como indicativo de um episódio maníaco iminente. Aparecem relatos de sensação de perda de controle em relação ao modo como os outros interpretam suas atitudes, o que pode ser inibitório para sua vida cotidiana e reforçar sentimentos comuns de insegurança a respeito de uma recaída e o aparecimento de um novo episódio de mania.

"Ah, assim, eu pensava que ficava alegre, ficava toda alegre, só que depois eu via que não estava bem, que eu estava mesmo era doente." (P2)

"Tenho medo da minha doença. Tenho medo porque qualquer hora pode acontecer outra loucura... Uma hora pode me dar uma coisa e eu não vejo o que eu faço. Igual a muitas coisas que eu já fiz..." (P1)

A incerteza quanto ao seu real estado de saúde mental pode estabelecer relações de dependência da opinião dos outros sobre si, sobretudo sobre seu estado mental. É como se, para esses pacientes com TAB, os outros pudessem saber mais do que eles próprios e que são capazes de ver neles coisas que eles mesmos não podem ver.

"Tinha meu tio pra vigiar pra não fazer loucura." (P1)

"Minha mãe uma vez falou que achava que eu não batia muito bem da cabeça." (P7)

\section{(3) Episódios maníacos estruturando relações interpessoais versus projeções da angústia}

O que foi vivenciado durante o episódio maníaco parece ser utilizado na fase de remissão para um reposicionamento pessoal diante do mundo ou como fonte de projeções de 
suas necessidades internas no ambiente. Aparecem angústia e sentimentos de rejeição, que podem desencadear vivências depressivas importantes.

"Se eu tivesse um amigo louco também me afastaria." (P1)

"(...) meus amigos me olhando, assim, com medo (...) eles falam que é loucura, que é besteira, que não existe, sei lá (...) ficavam com dó, com pena de ver o jeito que eu estava." (P1)

Quando sua autocrítica é muito intensa, gerando angústia, podem projetá-la nas pessoas que os rodeiam. Assim, os pacientes com TAB sentem-se julgados, rotulados e rejeitados, o que pode recrudescer sentimentos depressivos e de insegurança. Esse mecanismo de projeção pode, em parte, explicar a percepção que têm de que seus familiares, que aparecem nos relatos como os que os julgavam pejorativamente, criticavam e não compreendiam sua enfermidade. Percebem o seu problema como um fardo a ser carregado pela família e por si mesmos.

"Minha irmã fica 'pára que isso não tá funcionando', mesmo ela sendo agressiva, eles tentam ajudar sim, mas não é o ideal." (P4)

"É um fardo pra minha mãe, pra minha família, nossa, pra todos. Até para os meus amigos." (P1)

"(...) a turma se fazia de amigo, só que agora eu entendo bem (...) é companheiro de trabalho, mas já não confio mais, não sou, tipo, a postura que eu era antes, confiava demais nas pessoas... Hoje em dia eu já vejo. Pessoa está falando alguma coisa de mim ou falando mal de outro amigo (...) penso muito antes de dar uma resposta." (P7)

Os participantes relataram também diferentes crenças em relação à origem do transtorno, mas sempre ligadas a algum fator externo. Uma delas apontando para as relações sociais como geradoras dos episódios de mania.

"(...) não sei se os médicos acreditam, eu acho que pode ter sido alguma coisa maligna que alguém fez." (P1)

"Eu acho assim, porque o inimigo não conseguiu me levar da primeira vez, da segunda ele ia querer vir arrebentando mesmo (...)" (P7)

"Eu fiquei assim por causa de um namorado que eu achava que tinha largado de mim." (P2)

Ao perceberem que esses episódios acontecem a sua revelia e até mesmo sem que eles percebam, a busca por uma explicação racionalizada que possa dar um fim a esse sofrimento parece constante. Podem-se identificar alguns movimentos de projeção da angústia de perder o controle e passam a reavaliar suas relações, tentando identificar nelas as causas do seu transtorno.

"Deus quis assim, que eu herdasse essa doença do meu avô." (P1)
A religiosidade também aparece como um elemento externo para criar explicações para a origem dos episódios. Esta funcionaria como uma justificativa e, assim, evitaria qualquer contato com a perda do controle desses pacientes no processo do adoecimento.

"Se eu contar pro senhor... Olha, foi pra mim... O que
eu sofri... Hoje recebi uma graça." (P5)

"Eu penso, primeiramente, em Deus. Porque sei que ele tá acima de mim e que está me ajudando." (P3)

Demonstraram também a necessidade de um alívio do medo da ocorrência de novos episódios maníacos ao recorrer à religiosidade como fator protetor. Esse movimento pode ser entendido como uma aceitação possível de sua falta de controle sobre seu comportamento durante o quadro de mania, como uma forma de lidar com o medo de perda de controle.

\section{DISCUSSÃO}

Os pacientes com TAB entrevistados na presente pesquisa demonstraram sentimentos de ambivalência e vergonha, o que sugere que a ressignificação pessoal do que foi vivido, com a finalidade de elaboração, ficou barrada pela incapacidade de lidar com o que fez durante os episódios maníacos. Desse modo, um constrangimento foi identificado como obstáculo para a elaboração das vivências daquela fase. A vergonha aparece como uma marca do contraste entre o que essas pessoas fizeram nos momentos de crises maníacas e o que elas fazem no momento atual, com a sensação de perda de controle. De acordo com o estudo de Highfield et al. ${ }^{15}$, pacientes com TAB apresentam alto nível de sentimentos de vergonha e sugerem que os clínicos devem considerar a vergonha como um fator para a exploração em settings terapêuticos.

Assim, o episódio maníaco aparece nos relatos dos entrevistados como um estigma social e entende-se esse fenômeno no sentido que é dado por Goffman ${ }^{16}$, que descreve um estigma como sendo a situação de incapacidade de pertencer aos padrões normalizados da sociedade. É nesse sentido que se pode entender que os episódios maníacos provocam marcas na identidade dessas pessoas. Esses achados estão em concordância com os de Delmas et al. ${ }^{17}$, que assinalam que os pacientes com TAB aceitariam melhor o diagnóstico se houvesse maior conscientização da sociedade e diminuição da estigmatização associada ao transtorno, com maior apoio da família e dos amigos.

Os participantes da presente pesquisa relataram prejuízos nas suas relações considerando uma vivência de autoestigmatização, assim como os achados da revisão de Latalova et al. ${ }^{18}$, que evidenciam a presença de autoestigma nos pacientes com TAB. Esses autores constataram que tanto os pa- 
cientes quanto suas famílias são estigmatizados, e que essa estigmatização afeta a qualidade de vida e o funcionamento social dessas pessoas. Em decorrência disso, o suporte psicológico aos familiares e cuidadores é indicado nos tratamentos propostos para $T A B^{2,19}$.

O envolvimento de familiares para ajudar os pacientes a reconhecer os sinais precoces de mania ou depressão é um complemento útil para tratamento farmacológico e uma abordagem eficaz para a prevenção de recaída ${ }^{19,20}$, devendo ser mais explorado nas pesquisas científicas. A associação do estigma com dificuldades em relação ao tratamento é descrita na literatura ${ }^{18}$, o que está de acordo com os achados das entrevistas.

Os relatos também sugerem que, após a remissão do episódio, há projeção da angústia nas relações interpessoais, o que pode se associar aos achados de Tonelli21 , em relação à existência de possíveis prejuízos nos pacientes com TAB em compreender, afetiva e cognitivamente, as outras pessoas quanto à tomada de perspectiva sobre crenças, desejos e intenções. Essa percepção de estados mentais deficitária facilitaria a criação de falsas crenças sobre as reais intenções, pensamentos e desejos dos outros, repercutindo na forma com a qual se estabelecem seus relacionamentos interpessoais ${ }^{22}$.

De acordo com Vasconcelos et al..$^{23}$, os pacientes com TAB tendem a ser caracterizados como imaturos e dependentes, apresentando dificuldade para se responsabilizarem pelas consequências do próprio comportamento de acordo com suas escolhas. Isso parece estar relacionado com as tentativas de encontrar explicações no ambiente, e não em si mesmos. Os relatos dos entrevistados, que atribuíam a causa de seus episódios de mania a terceiros, são corroborados com os achados de Hosang et al. ${ }^{24}$, cujos participantes relataram que um problema sério com um amigo, vizinho ou parente antecedeu ao pior episódio maníaco que tiveram. Os participantes da presente pesquisa também relataram associação entre problemas nas relações interpessoais e o desencadeamento de episódios maníacos.

Os participantes do presente estudo também se utilizam de atribuições religiosas como justificativa externa para explicar a origem dos episódios, associação essa já relatada na literatura ${ }^{25,26}$. Eles parecem utilizar a temática religiosa a fim de minimizar as repercussões das fases de mania, aliviando a sensação angustiante da falta de controle sobre suas reincididas, o que se contrapõe aos achados descritos por Mitchell e Romans ${ }^{27}$, cujos pacientes relatavam que suas crenças não os ajudavam a lidar com o transtorno.

Assim, encontram-se desdobramentos positivos e negativos do papel da religiosidade na vida desses pacientes. Os achados de Stroppa e Moreira-Almeida ${ }^{25}$ sugerem que a religiosidade e a espiritualidade nos pacientes com $T A B$ podem ser utilizadas como estratégias de coping positivas que resultam em bem-estar, confiança e calma. Porém, os autores também apontam para repercussões negativas que podem gerar culpa, medo e autodesvalorização, e ressaltam a importância de apoio psicoeducacional para orientar os pacientes em relação à religiosidade.

Em associação aos achados, sugere-se então que a questão da religiosidade e espiritualidade deva ser considerada caso a caso, diante da possibilidade de ter um papel impeditivo na elaboração dos episódios maníacos, funcionando como uma fonte de mais angústia e projeções ou facilitador como um suporte para lidar com o transtorno.

Da mesma forma, devem-se considerar os relatos de associação de fatores externos que interferiram em um episódio maníaco na perspectiva do paciente, numa tentativa de conscientizá-lo acerca de suas próprias contribuições e da interpretação que fizeram dos acontecimentos de suas vidas. A finalidade dessa análise seria, então, de não interromper o processo de elaboração dos episódios de mania com uma correlação causal, mas possibilitar um processo de pensar sobre o seu transtorno de uma forma mais efetiva para uma real elaboração do que foi vivido e uma consequente transformação na sua capacidade de lidar com o transtorno.

As limitações da presente pesquisa estão no fato de que a análise apresentada neste artigo é a interpretação dos pesquisadores envolvidos em relação ao que os participantes relataram sobre suas perspectivas quanto aos episódios de mania. Além disso, por se tratar de uma pesquisa realizada em um ambulatório comum a todos os entrevistados, fazem-se necessárias investigações científicas em outros serviços de outras localidades, a fim de correlacionar dados sociodemográficos aos achados.

A presente pesquisa foi abrangente em relação aos tópicos de discussão propostos, o que também pode ser entendido como uma das limitações desse estudo. Desse modo, para as próximas pesquisas, podem ser feitos recortes mais específicos, como abordar a questão das relações com familiares a fim de estabelecer condutas eficientes no manejo dos episódios e/ou na prevenção deles para orientá-los no apoio ao tratamento.

Os resultados podem fornecer subsídios a médicos e demais profissionais de saúde para melhor manejar estratégias de aderência dos pacientes aos tratamentos medicamentosos e para as medidas terapêuticas e preventivas da recorrência dos episódios. Os resultados deste trabalho podem, ainda, ser utilizados nas instituições para melhorar a relação médico-paciente, considerando que a projeção, aqui identificada, pode acontecer também na figura do médico e/ou na equipe multiprofissional envolvida.

\section{CONCLUSÃO}

Com base no aprofundamento da análise dos significados dos episódios maníacos relatados pelos pacientes em remissão, os achados da presente pesquisa contribuem para maior compreensão dos quadros maníacos no TAB. Observou-se que sentimentos como vergonha e medo e correlações cau- 
sais podem impedir elaborações a respeito do que foi vivido nos episódios de mania e que a equipe multiprofissional envolvida no tratamento desses casos precisa estar instrumentalizada para lidar com tais elementos e criar um espaço de confiança e acolhimento para receber essa demanda.

\section{CONTRIBUIÇÕES INDIVIDUAIS}

Luiz Carlos Pereira Bin - Contribuiu na concepção e desenho do estudo, na análise e interpretação dos dados e na elaboração do artigo.

Lia Keuchguerian Silveira Campos - Contribuiu na análise e interpretação dos dados, na elaboração do artigo e na revisão crítica de todo o conteúdo intelectual.

Amilton dos Santos Junior - Contribuiu na análise e interpretação dos dados, na elaboração do artigo e na revisão crítica de todo o conteúdo intelectual.

Egberto Ribeiro Turato - Contribuiu na concepção e desenho do estudo, na análise e interpretação dos dados e na revisão crítica de todo o conteúdo intelectual. A versão final foi aprovada por todos os autores.

\section{CONFLITOS DE INTERESSE}

Não há conflitos de interesse.

\section{AGRADECIMENTOS}

Os pesquisadores, em especial o primeiro autor, agradecem à bolsa da Fundação de Amparo à Pesquisa do Estado de São Paulo (Fapesp).

\section{REFERÊNCIAS}

1. Sadock BJ, Sadock VA. Kaplan \& Sadock Compêndio de psiquiatria: ciências do comportamento e psiquiatria clínica. 9a ed. Porto Alegre: Artmed; 2007.

2. World Health Organization (WHO). Chapter V - Mental and behavioural disorders (FOOF99) Mood [affective] disorders (F30-F39) 2007 Updated. Geneva: World Health Organization. Disponível em: <http://apps.who.int/classifications/apps/icd/icd10online2007/ index.htm?gf50.htm>. Acesso em: 26 mar. 2014.

3. Moreno RA, Moreno DH, Ratzke R. Diagnóstico, tratamento e prevenção da mania e da hipomania no transtorno bipolar. Rev Psiq Clín. 2005;32(Supl 1):39-48.

4. Silva RA, Mograbi DC, Silveira LAS, Nunes ALS, Novis FD, Cavaco PA, et al. Mood selfassessment in bipolar disorder: a comparison between patients in mania, depression, and euthymia. Trends Psychiatry Psychother. 2013;35(2):141-5.
5. Souza FGM. Tratamento do transtorno bipolar: eutimia. Rev Psiq Clín. 2005;32(1):63-70.

6. Novis F, Cheniaux E, Landeira-Fernandez J, Versiani M. A five year follow-up study of 11 patients with bipolar disorder. J Bras Psiquiatr. 2010;59(3):219-22.

7. Abreu LN, Lafer B, Baca-Garcia E, Oquendo MA. Suicidal ideation and suicide attempts in bipolar disorder type l: an update for the clinician. Rev Bras Psiquiatr. 2009;31(3):271-80.

8. Caycedo BML, Herrera S, Offir-Jiménez JK. Descripción de los factores de riesgo para recaída en pacientes con diagnóstico de trastorno mental. Rev Cienc Salud. 2011;9(2):141-58.

9. Hosang GM, Korszun A, Jones L, Jones I, Gray JM, Gunasinghe CM, et al. Adverse life event reporting and worst illness episodes in unipolar and bipolar affective disorders: measuring environmental risk for genetic research. Psychol Med. 2010;40:1829-37.

10. Turato ER. Tratado da metodologia da pesquisa clínico-qualitativa. Construção teóricoepistemológica, discussão comparada e aplicação nas áreas da saúde e humanas. 5 a ed. Petrópolis: Vozes; 2011

11. Turato ER. Métodos qualitativos e quantitativos na área da saúde: definições, diferenças e seus objetos de pesquisa. Rev Saude Publica. 2005;39(3):507-14.

12. Fontanella BJB, Ricas J, Turato ER. Amostragem por saturação em pesquisa qualitativa em saúde: contribuiçōes teóricas. Cad Saude Publica. 2008;24:17-27.

13. Fontanella BJB, Campos CJG, Turato ER. Data collection in clinical-qualitative research use of non-directed interviews with open-ended questions by health professionals. Rev Latino-am Enfermagem. 2006;14(5):812-20.

14. Campos CJG, Turato ER. Content analysis in studies using the clinical-qualitative method: application and perspectives. Rev Latino-am Enfermagem. 2009;17(2):259-64.

15. Highfield J, Markham D, Skinner M, Neal A. An investigation into the experience of selfconscious emotions in individuals with bipolar disorder, unipolar depression and nonpsychiatric controls. Clin Psychol Psychother. 2010;17:395-405.

16. Goffman E. Estigma: notas sobre a manipulação da identidade deteriorada. Rio de Janeiro: Zahar; 1982.

17. Delmas K, Proudfoot J, Parker G, Manicavasagar V. Recording past experiences: a qualitative study of how patients and family members adjust to the diagnosis of bipolar disorder. J Nerv Ment Dis. 2012;200(11):920-3.

18. Latalova K, Ociskova M, Prasko J, Kamaradova D, Jelenova D, Sedlackova Z. Self-stigmatization in patients with bipolar disorder. Neuro Endocrinol Lett. 2013;34(4):265-72.

19. National Institute for Health and Clinical Excellence. The management of bipolar disorder in adults, children and adolescence, in primary and secondary care. London: NICE; 2006. NICE Clinical Guideline 38. <http://guidance.nice.org.uk/CG38> .

20. Peters S, Pontin E, Lobban F, Morriss R. Involving relatives in relapse prevention for bipolar disorder: a multi-perspective qualitative study of value and barriers. BMC Psychiatry. 2011;11:172-82

21. Tonelli HA. Processamento cognitivo "Teoria da Mente" no transtorno bipolar. Rev Bras Psiquiatr. 2009;31(4):369-74.

22. Tonelli HA. Empatia no transtorno afetivo bipolar. Rev Psiq Clín. 2011;38(5):207-8

23. Vasconcelos AG, Malloy-Diniz LF, Nascimento E, Neves F, Corrêa H. Traços de temperamento associados ao transtorno afetivo bipolar: uma revisão integrativa da literatura. Trends Psychiatry Psychother. 2011;33(3):169-80.

24. Hosang GM, Korszun A, Jones L, Jones I, McGuffin P, Farmer AE. Life-event specificity: bipolar disorder compared with unipolar depression. Br J Psychiatry. 2012;201(6):458-65.

25. Stroppa A, Moreira-Almeida A. Religiosidade e espiritualidade no transtorno bipolar do humor. Rev Psiq Clín. 2009:36(5):190-6.

26. Dantas $(R$, Pavarin $L B$, Dalgalarrondo P. Sintomas de conteúdo religioso em pacientes psiquiátricos. Rev Bras Psiquiatr. 1999;21(3):158-64.

27. Mitchell L, Romans S. Spiritual beliefs in bipolar affective disorder: their relevance for illness management. J Affect Disord. 2003;75:247-57. 